\title{
New nidus formation adjacent to the target site of an arteriovenous malformation treated by Gamma Knife surgery
}

\author{
Report of 3 cases \\ Jung-Ho Yun, M.D., Do Hoon Kwon, M.D., Ph.D., Eun Jung Lee, M.D., \\ Do Heui Lee, Ph.D., Jae Sung Ahn, M.D., Ph.D., And Byung Duk Kwun, M.D., Ph.D. \\ Department of Neurological Surgery, Asan Medical Center, College of Medicine, University of Ulsan, \\ Seoul, Korea
}

\begin{abstract}
New nidi are rarely found adjacent to the resection margin following treatment for an arteriovenous malformation (AVM), especially in adults. In addition, there are no reports in adults of new nidus formation adjacent to the targeted site of an AVM that angiography has verified to be completely obliterated by radiosurgery. The authors present their experience with recurrent AVMs following AVM radiosurgery in 3 patients whose ages were 9 years, 10 years, and 33 years. None of the patients had been treated with embolization before radiosurgery. Two patients had a history of intracerebral hemorrhage before radiosurgery. New lesions developed around the obliterated nidi in all 3 cases. Angiography performed after the first radiosurgery confirmed complete removal of the nidus in all 3 patients, and new nidus formation was detected 31,132, and 36 months after the initial GKS. The new lesions were also treated by GKS. Occasionally, in patients with recurrent AVMs, such as those described in this paper, long-term clinical and angiographic follow-up may be required, even if complete occlusion is originally shown on angiograms. (http://thejns.org/doi/abs/10.3171/2012.8.GKS12994)
\end{abstract}

$\begin{array}{llrl}\text { KEY WoRDS } & \bullet \text { nidus } & \bullet & \text { arteriovenous malformation } \\ \text { stereotactic radiosurgery } & \bullet \quad \text { Gamma Knife surgery }\end{array}$

$\mathrm{C}$ OMPLETE resection or total obliteration of cerebral AVMs after GKS is generally believed to eliminate any further risk of hemorrhage. According to the literature, large series with long-term surgical results following resection of cerebral AVMs have shown that patients in whom postoperative angiograms show no evidence of lesions do not suffer repeated hemorrhage. ${ }^{3}$ Cases of recurrent AVM have been reported after gross-total microsurgical removal, but this is very rare, especially in the adult population. To date, recurrent AVMs in adults after complete occlusion of the nidus following radiosurgery has not been reported. In this article, we present our experience with 3 patients in whom new nidi developed near the targeted site of an AVM that had been completely occluded by radiosurgery. We performed a retrospective analysis of 1152 AVM cases treated with GKS at our institution during a 21-year period. Among these, 3 cases were found to have new nidus formation adjacent to the

Abbreviations used in this paper: AVM = arteriovenous malformation; GKS = Gamma Knife surgery; ICH = intracerebral hemorrhage; VEGF = vascular endothelial growth factor. targeted lesion at 31, 132, and 36 months after an initial GKS had completely occluded the lesions, as verified on angiographic studies.

Case 1

\section{Case Reports}

The first patient was a 9-year-old boy treated by GKS for a left occipital AVM 1 month after he experienced $\mathrm{ICH}$. The treated volume was $600 \mathrm{~cm}^{3}$, and 25 Gy was directed to the margin of the nidus. Follow-up angiography performed 1 year after radiosurgery showed a decreased size of the nidus at the same location. Thirty-one months after the initial GKS, follow-up angiography showed that the targeted nidus had been completely obliterated, but new nidus formation was noted inferior to the earlier lesion. We therefore performed a second GKS to treat this new nidus, and the cumulative treated volume increased to $900 \mathrm{~cm}^{3}$ (Fig. 1). On cerebral angiographic studies, the new lesion completely disappeared 2 years after the second radiosurgery. Meticulous clinical follow-up examinations continue to be performed in this patient for the detection of an asymptomatic AVM recurrence. 

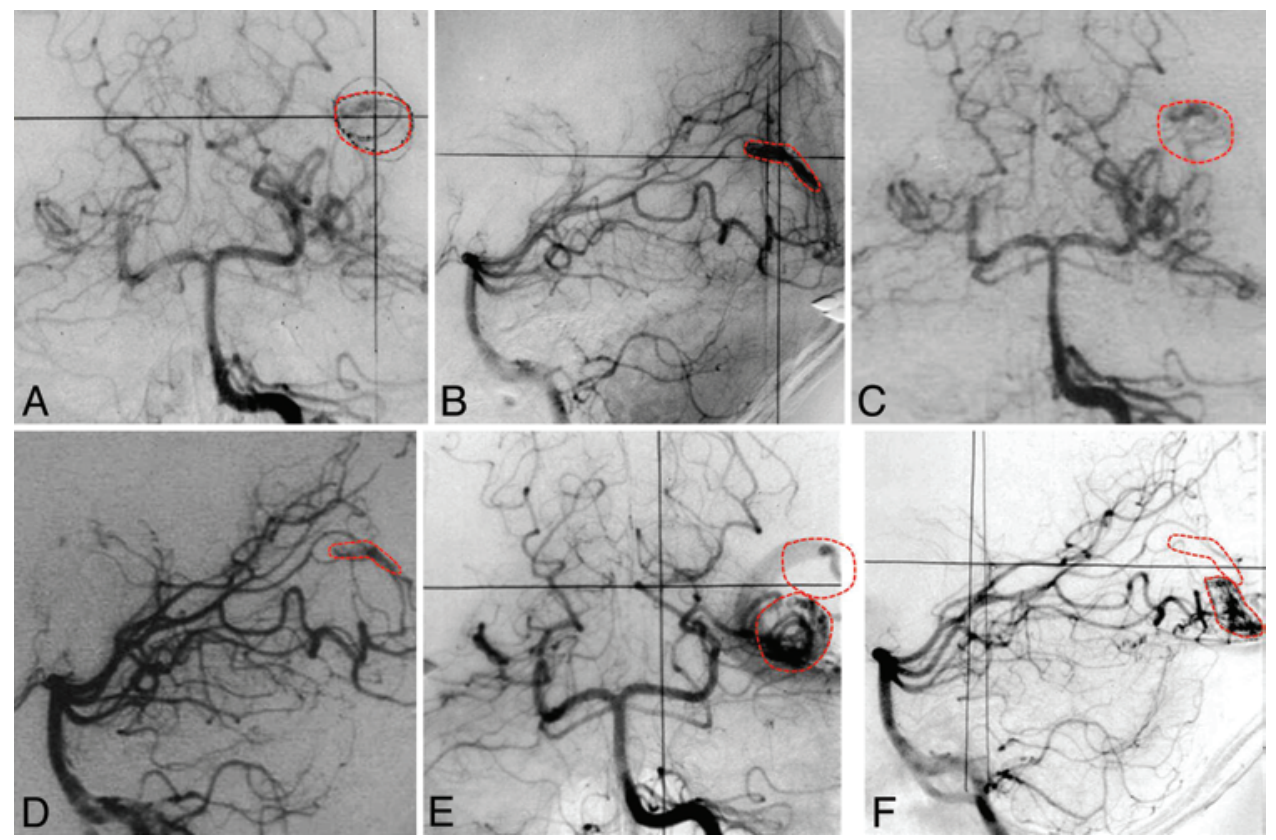

Fig. 1. Case 1. Vertebral angiogram obtained before (A and B), 1 year (C and D), and 31 months (E and $F$ ) after the original radiosurgery. During that procedure, anteroposterior $(A)$ and lateral $(B)$ angiographic images were obtained, confirming the initial nidus (circle). One year after radiosurgery, a partially occluded small nidus ( $C$ and $D$ ) is still noted. A new nidus (lower circle) was noted inferior to the previous nidus (upper circle) 31 months after the original radiosurgery ( $E$ and $F$ ). Repeated radiosurgery was performed for this new nidus.

\section{Case 2}

The second patient was a 10-year-old boy treated by GKS for a small AVM located in the cerebellar vermis 1 month after he initially presented with bleeding. The treated volume was $700 \mathrm{~cm}^{3}$ (Fig. 2A and Fig. 3A and D). Five years after radiosurgery, the patient suffered from repeated hemorrhage at the same site, and angiography revealed a partially occluded nidus with a total volume of $400 \mathrm{~cm}^{3}$. The patient underwent GKS 2 months after the repeated hemorrhage with the radiation dose directed to the same nidus (Fig. 2B and Fig. 3B and E). Six years after the second treatment, routine MR imaging revealed a new lesion located medial to the previously targeted nidus, which was finally completely occluded (Fig. 2C). Follow-up angiography showed the volume of this new nidus to be $2800 \mathrm{~cm}^{3}$ (Fig. 3C and F). A third GKS was successfully performed to treat the new nidus. Two years later, MR angiography confirmed that the new lesion had been completely obliterated (Fig. 2D). We plan to perform an additional angiographic study 5 years after the last radiosurgery to check for an asymptomatic recurrent AVM.

\section{Case 3}

The third patient was a 33-year-old man treated with GKS for an incidentally detected cerebellar AVM whose nidus volume was $1100 \mathrm{~cm}^{3}$. A radiation dose of $20 \mathrm{~Gy}$ was delivered to the lesion's margin (Fig. 4A and B). Three years after radiosurgery, the patient experienced a cerebellar ICH and angiography revealed a new nidus adjacent to the previously targeted lesion, which was completely occluded (Fig. 4C and D). Radiosurgery was repeated for this new lesion whose volume was $500 \mathrm{~cm}^{3}$. Unfortunately, we do not have additional information on the patient's response to the last GKS because he was lost to follow-up.

\section{Discussion}

\section{Literature Review}

Curative treatment of cerebral AVMs can be performed in a variety of ways: complete resection of abnormal vasculature, such as the nidus and arteriovenous shunting, by using a microsurgical procedure; total obliteration of the nidus by radiosurgery; or total embolization of the nidus using an endovascular procedure. Angiographic evidence of an absent nidus following these treatments generally indicates no further risk of hemorrhage; therefore, additional angiographic studies are not necessary. ${ }^{2,5,6,10,12,15,19,20,30}$ Recurrence after the disappearance of an AVM nidus has been reported occasionally, however, with 30 cases reported in the English-language literature. . $3,4,7,8,11,13,14,16-18,22,23,26,29,31$ Nevertheless only one pediatric case of recurrent AVM after radiosurgery has been reported.$^{24}$ In this article, we present three cases of AVM recurrence after successful GKS-one in an adult and two in children (Table 1). This is the first report of AVM recurrence in an adult after complete obliteration of the nidus following radiosurgery.

Kader et al. ${ }^{16}$ reported 5 pediatric cases of AVM recurrences in patients in whom postoperative angiograms yielded negative findings; in those cases, the recurrences appeared 1 to 9 years after the initial resection. Similarly, Klimo et al. ${ }^{17}$ described 5 pediatric cases of recurrent AVMs; in all these cases postoperative angiography had 

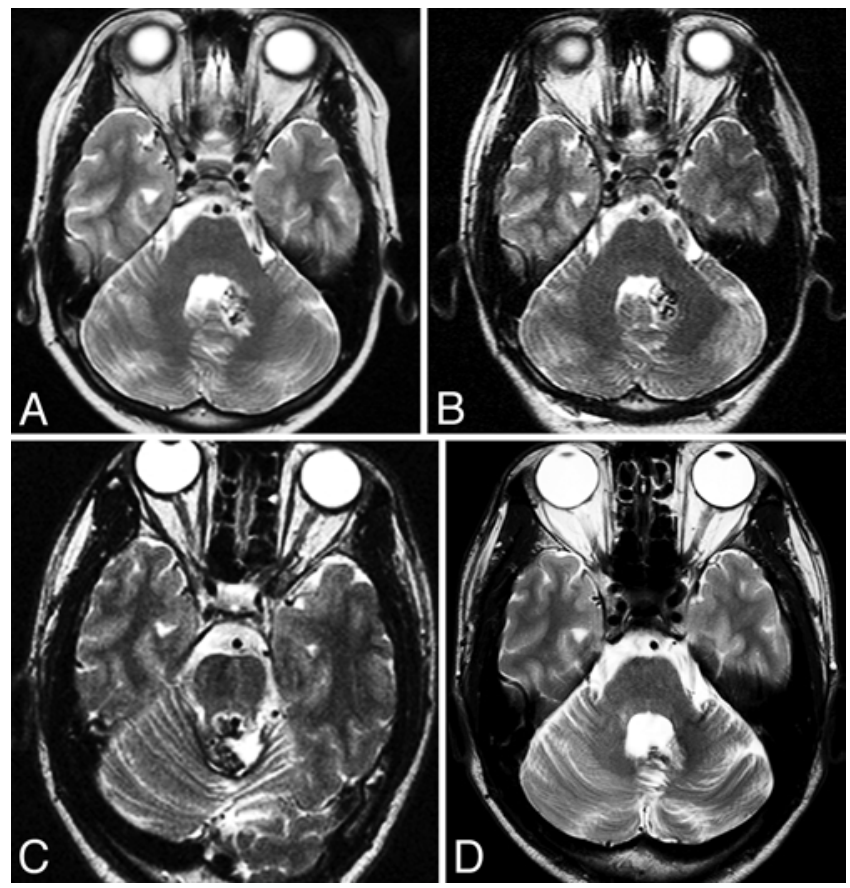

FIG. 2. Case 2. Axial T2-weighted MR images obtained 1 year (A), 5 years (B), 11 years (C), and 13 years (D) after radiosurgery. One year after radiosurgery, the nidus is still near the left side of the fourth ventricle (A). Five years after radiosurgery, the nidus remains at the same location, and a second radiosurgery is performed to treat this partially occluded nidus (B). Six years after the second radiosurgery, a new nidus is now observed at the midline vermis (C). A third GKS was successfully performed to treat the new nidus. Two years later, MRI confirmed that the new lesion had been completely obliterated (D).

confirmed that the original lesion had been completely resected. The authors reported that in all cases the AVM recurred within 6 years, including 1 patient who experienced 2 recurrences, and that the diffuse type of AVM was more likely to recur.

Although AVM recurrences after successful surgical or radiosurgical treatment have been reported in children, these events remain extremely rare in the adult population. Previously, only 5 adult cases of recurrent AVM with negative results on postoperative angiograms were reported in the literature. ${ }^{4,7,8,13,25}$ Gabriel et al. ${ }^{8}$ first reported an AVM recurrence in an adult after complete resection. The patient was a 19-year-old man with angiographic confirmation of complete AVM excision; recurrence occurred 9 years after the initial operation. The remaining four adults with AVM recurrences included 3 men and 1 woman ranging in age from 21 and 33 years. In these patients, the latency period between initial resection of the lesion and recurrence ranged from 4 to 9 years. Codd et al. ${ }^{4}$ presented the first adult case of double recurrence of an AVM in a 33-year-old woman with a Spezler-Martin Grade III AVM in the left occipital lobe. She experienced 2 recurrences, with the first occurring 7 years after initial complete resection and the second detected 2 years after the second resection. As an alternative treatment, stereotactic radiosurgery with an 18-Gy dose was performed.

We believe that we are the first to report an adult case in which the patient underwent radiosurgery, received confirmation of total obliteration of the nidus, and then experienced an AVM recurrence. We are reporting similar experiences in pediatric cases, but an AVM recurrence after radiosurgery has already been reported in a pediatric patient. Rodríguez-Arias et al. ${ }^{24}$ presented the case of a 9-year-old patient in whom an AVM recurred in a different location 2 years after GKS had eradicated the lesion with a $25-\mathrm{Gy}$ dose to the $75 \%$ isodose line. The authors suggested that the redistribution of blood flow after progressive occlusion of the AVM may have acted on immature vessels surrounding the malformation, giving rise to another AVM. These authors also proposed that repeated angiography at the beginning of adult life should be considered for successful and consistent exclusion of any recurrence of AVMs treated by radiosurgery in pediatric patients.

Pathogenesis of Recurrent AVMs After Operative Resection. Several reports in the literature suggest possible theories for the pathogenesis of recurrent AVMs after complete resection. Gabriel et al. ${ }^{8}$ proposed that a small residual nidus may be related to an AVM recurrence. In that case, these recurrences may not be true recurrences, but could trigger events that could conceal the nidus temporarily on angiographic studies. Factors associated with residual nidi, such as vessel spasm, brain swelling with a compressive mass effect, or temporary thrombosis of residual AVM vasculature in the immediate postoperative period, could explain the absence of the residual nidus on angiograms obtained immediately postoperatively.

Also, small immature AVM vessels, which are generally detected in pediatric cases, may be a potential factor in AVM regrowth. ${ }^{9}$ Although these vessels may appear invisible on angiograms, they may retain the ability to form new AVMs in the same location. Likewise, Padget ${ }^{21}$ presented a fistula model consisting of an immature aberrant vessel connection between the primitive arteries and relatively large veins occurring during childhood. It has been further hypothesized that reduced resistance to blood flow in such arteriovenous fistulas may cause abnormal arteries to dilate adjacent to normal arteries.

Another alternative theory is related to the angiogenesis stimulated by brain injury, tumor, ischemia, and/or inflammation. Schmit et al. ${ }^{27}$ hypothesized that localized ischemia and inflammation could induce AVM recurrence by hyperstimulating angiogenic factors, as suggested in a de novo AVM model in an 11-year-old patient with moyamoya. Sonstein et al. ${ }^{28}$ proposed that VEGF expression may be associated with regrowth of AVMs in both pediatric and adult patients. Furthermore, Kader et al. ${ }^{16}$ suggested that surgery itself, performed during an active growth stage in patients in whom proangiogenic tissue remains after resection, may produce an angiogenic process, potentially increasing the risk of recurrence. They also suggested that VEGF may play a role in the recurrence of AVMs. Previous reports by Hashimoto et al., ${ }^{11}$ showed that AVM vessels in pediatric patients had a tendency to have a high Ki-67 index. These results offer evidence of increased endothelial cell turnover in AVMs, which may be indicative of an active angiogenic process.

Another possible hypothesis to explain true AVM 


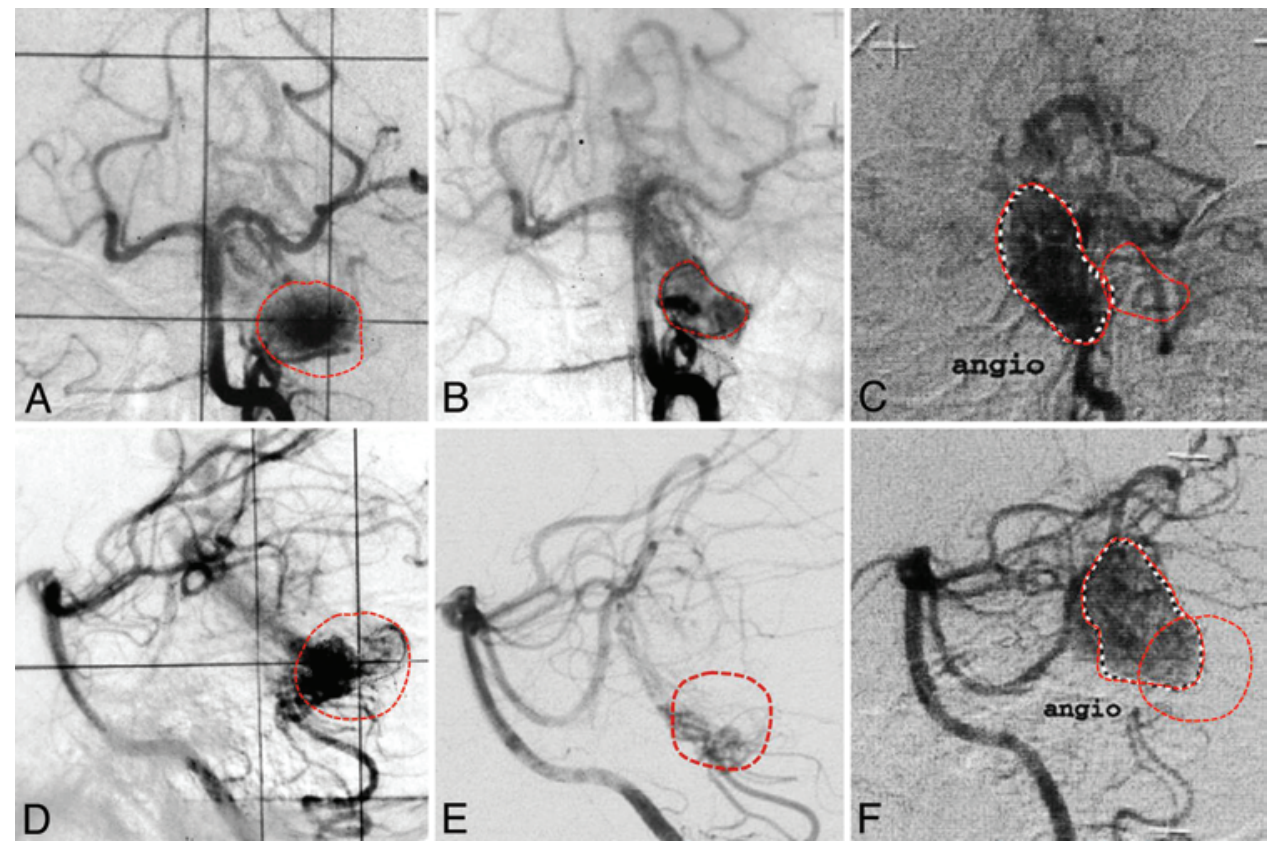

FIG. 3. Case 2. Vertebral angiograms obtained before and after radiosurgery. Anteroposterior (A) and lateral (D) angiograms revealing the initial AVM nidus (circle). Five years after radiosurgery, a small nidus is still noted on anteroposterior (B) and lateral (E) angiograms. Repeated radiosurgery was performed for this partially occluded lesion. Seventy-two months after the first radiosurgery, a new nidus (large circle) was noted superomedial to the initial nidus (small circle), which is shown to be completely occluded on anteroposterior $(C)$ and lateral $(F)$ angiograms. A third radiosurgery was performed to treat this new nidus.

recurrence is the "hidden compartment" model. ${ }^{23}$ This concept suggests that a change in the vascular supply, as shown by differences in the feeding artery between the initial and recurrent $\mathrm{AVM}$, could indicate the presence of multiple compartments. Each compartment could have feeding arteries, draining veins, and nidi that are separate from the excised unfilled compartment. Although unseen on early postoperative angiographic studies, the partially thrombosed sector may have a vessel that connects with vessels in other compartments. Similar to this hypothesis, there is the "reserve nidus" concept suggested by Sano et al., ${ }^{25}$ which is defined as an abnormal vascular group adjacent to the primary nidus, which subsequently grows to become a recurrent lesion capable of rebleeding.

Possible Pathogenesis of Recurrent AVMs in Radiosurgery Cases in This Series. These aforementioned hypotheses could be applied to the recurrence of an AVM after total occlusion of the nidus following radiosurgery, such as that seen in the present cases. Although we cannot fully explain the factors related to AVM recurrence after radiosurgery, we can consider that the cause of the recurrence is the redistribution of blood flow after progressive occlusion of the AVM, as suggested by Rodríguez-Arias et al. ${ }^{24}$ In addition, we cannot completely exclude a new theory that suggests that radiation can induce angiogenesis of the surrounding nidus, in contrast to the previous concept that radiation inhibits angiogenesis of the AVM nidus. This theory is supported by the present study, in which all three patients had recurrence adjacent to the targeted site of the AVM. The lesion was located in the previous margin area and could have been affected by the radiation effect of the previous radiosurgery.
In our first case, the patient was treated with radiosurgery 1 month after experiencing hemorrhage. Because the new nidus was noted adjacent to the previous nidus, we could hypothesize that there was reexpansion of a hidden

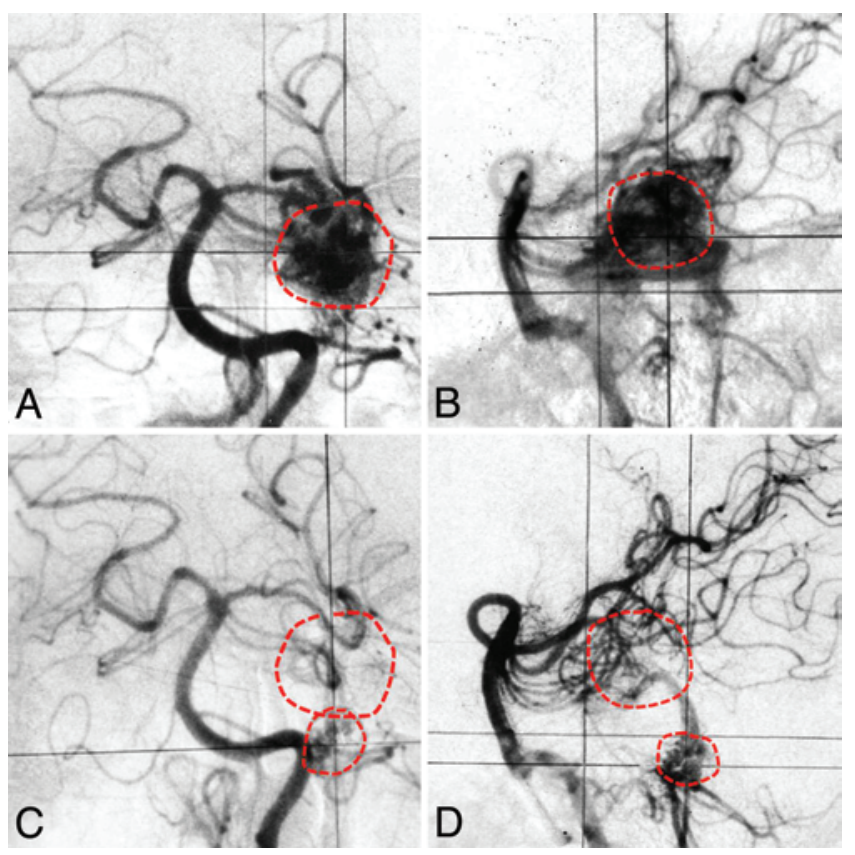

FIG. 4. Case 3. Vertebral angiograms including anteroposterior (A) and lateral $(\mathbf{B})$ views obtained before radiosurgery. Three years after radiosurgery, a new nidus (smaller circle) was noted inferior to the previous target (larger circle), which was shown to be completely occluded on anteroposterior (C) and lateral (D) angiograms. A second radiosurgery was performed for this new nidus. 
TABLE 1: Characteristics in 3 patients with AVM recurrence*

\begin{tabular}{|c|c|c|c|c|c|c|c|c|c|}
\hline \multirow[b]{2}{*}{$\begin{array}{l}\text { Case } \\
\text { No. }\end{array}$} & \multirow[b]{2}{*}{$\begin{array}{l}\text { Age (yrs), } \\
\text { Sex }\end{array}$} & \multirow[b]{2}{*}{ Initial Sx } & \multirow[b]{2}{*}{ AVM Location } & \multicolumn{2}{|c|}{ Initial AVM } & \multicolumn{2}{|c|}{ Recurred AVM } & \multirow[b]{2}{*}{$\begin{array}{c}\text { Time to } \\
\text { Recurrence (mos) }\end{array}$} & \multirow[b]{2}{*}{ Results at Last FU } \\
\hline & & & & Size $\left(\mathrm{cm}^{3}\right)$ & $\begin{array}{c}\text { Dose, } \\
\text { Isodose Line }\end{array}$ & Size $\left(\mathrm{cm}^{3}\right)$ & $\begin{array}{c}\text { Dose, } \\
\text { Isodose Line }\end{array}$ & & \\
\hline 2 & $10, M$ & $\mathrm{ICH}$ & LCBM & $\begin{array}{l}700 \\
400\end{array}$ & $\begin{array}{l}25 \mathrm{~Gy}, 70 \% \\
25 \mathrm{~Gy}, 50 \%\end{array}$ & 2800 & 23 Gy, 50\% & 72 & nidus absent on MRA \\
\hline 3 & $33, M$ & none & LCBM & 1100 & 20 Gy, $50 \%$ & 500 & $25 \mathrm{~Gy}, 70 \%$ & 36 & LOF \\
\hline
\end{tabular}

* FU = follow-up; LCBM = left cerebellum; LOF = lost to follow-up; LOL = left occipital lobe; MRA = MR angiography; Sx = symptoms; TFCA = transfemoral cerebral angiography.

compartment compressed by a hematoma. However, on the follow-up angiograms obtained 1 year after radiosurgery, the nidus volume was smaller and no new lesion was detected around the targeted nidus. Then 31 months after radiosurgery, a new nidus appeared adjacent to the targeted lesion, which had been completely occluded. In this case, the theory of reexpansion of a residual compartment, such as a nidus compressed by hematoma, can be discarded and the theory of a true AVM recurrence may apply. In the second case, a newly enlarged AVM was noted adjacent to the previous nidus after 2 rounds of radiosurgery. After the first radiosurgery, the patient experienced rebleeding at the same location due to partial occlusion of the AVM. After the second radiosurgery, the patient exhibited no symptoms, but a new nidus was noted 11 years after the first radiosurgery, with a volume larger than that of the initial nidus. The possibility of reexpansion of the nidus that had been compressed by a hematoma can be discarded because of the long-term follow-up interval. Also, the significantly larger volume of the new AVM, compared with that of the previous target volume, does not fit into the theory of residual nidus expansion. In the third case, the patient had no ICH at the initial radiosurgery, and the targeted nidus was completely occluded after radiosurgery. However, ICH inferior to the previous target developed 3 years after radiosurgery. This case also presents as the development of a new nidus, because there was no possibility of reexpansion of the compressed nidus.

On the basis of our analysis, we believe that our three cases are "true" AVM recurrences for several reasons. First, postradiosurgery angiograms demonstrated no obvious spasm and no thrombosis that potentially could have concealed a residual nidus. Second, the time interval between radiosurgery and follow-up angiography was long enough to recover from the pathological conditions mentioned earlier. Finally, we did not perform embolization for these AVMs, which could have led to recanalization of the initial nidus.

\section{Conclusions}

The present cases suggest that a new nidus can be found adjacent to the target site of an AVM after GKS. In addition, we describe the first adult case of AVM recurrence after complete occlusion was verified by postradiosurgery angiography. Our cases document that angiographic confirmation of total absence of the nidus does not always eliminate the risk of AVM regeneration with the potential of rebleeding, even in adults. Therefore, some patients whose AVMs have been eliminated following resection or radiosurgery, such as the patients presented in this paper, may require long-term clinical and angiographic follow-up.

\section{Disclosure}

The authors report no conflict of interest concerning the materials or methods used in this study or the findings specified in this paper.

Author contributions to the study and manuscript preparation include the following. Conception and design: Kwon, Yun. Acquisition of data: Kwon, EJ Lee. Analysis and interpretation of data: Yun. Drafting the article: Yun. Critically revising the article: all authors. Reviewed submitted version of manuscript: all authors. Approved the final version of the manuscript on behalf of all authors: Kwon. Administrative/technical/material support: Yun, Ahn, DH Lee, EJ Lee. Study supervision: Kwon, Kwun, Ahn, DH Lee.

\section{References}

1. Ali MJ, Bendok BR, Rosenblatt S, Rose JE, Getch CC, Batjer $\mathrm{HH}$ : Recurrence of pediatric cerebral arteriovenous malformations after angiographically documented resection. Pediatr Neurosurg 39:32-38, 2003

2. Amacher AL, Allcock JM, Drake CG: Cerebral angiomas: the sequelae of surgical treatment. J Neurosurg 37:571-575, 1972

3. Andaluz N, Myseros JS, Sathi S, Crone KR, Tew JM Jr: Recurrence of cerebral arteriovenous malformations in children: report of two cases and review of the literature. Surg Neurol 62:324-331, 2004

4. Codd PJ, Mitha AP, Ogilvy CS: A recurrent cerebral arteriovenous malformation in an adult. J Neurosurg 109:486-491, 2008

5. Davis C, Symon L: The management of cerebral arteriovenous malformations. Acta Neurochir (Wien) 74:4-11, 1985

6. Drake CG: Cerebral arteriovenous malformations: considerations for and experience with surgical treatment in 166 cases. Clin Neurosurg 26:145-208, 1979

7. Freudenstein D, Duffner F, Ernemann U, Rachinger J, Grote $\mathrm{E}$ : Recurrence of a cerebral arteriovenous malformation after surgical excision. Cerebrovasc Dis 11:59-64, 2001

8. Gabriel EM, Sampson JH, Wilkins RH: Recurrence of a cerebral arteriovenous malformation after surgical excision. Case report. J Neurosurg 84:879-882, 1996

9. Gallina P, Merienne L, Meder JF, Schlienger M, Lefkopoulos $\mathrm{D}$, Merland JJ: Failure in radiosurgery treatment of cerebral arteriovenous malformations. Neurosurgery 42:996-1004, 1998 
10. Guidetti B, Delitala A: Intracranial arteriovenous malformations. Conservative and surgical treatment. J Neurosurg 53: $149-152,1980$

11. Hashimoto N, Nozaki K: Do cerebral arteriovenous malformations recur after angiographically confirmed total extirpation? Crit Rev Neurosurg 9:141-146, 1999

12. Heros RC, Korosue K, Diebold PM: Surgical excision of cerebral arteriovenous malformations: late results. Neurosurgery 26:570-578, 1990

13. Hino A, Fujimoto M, Iwamoto Y, Takahashi Y, Katsumori T: An adult case of recurrent arteriovenous malformation after "complete" surgical excision: a case report. Surg Neurol 52: $156-159,1999$

14. Hladky JP, Lejeune JP, Blond S, Pruvo JP, Dhellemmes P: Cerebral arteriovenous malformations in children: report on 62 cases. Childs Nerv Syst 10:328-333, 1994

15. Jomin M, Lesoin F, Lozes G: Prognosis for arteriovenous malformations of the brain in adults based on 150 cases. Surg Neurol 23:362-366, 1985

16. Kader A, Goodrich JT, Sonstein WJ, Stein BM, Carmel PW, Michelsen WJ: Recurrent cerebral arteriovenous malformations after negative postoperative angiograms. J Neurosurg 85:14-18, 1996

17. Klimo P Jr, Rao G, Brockmeyer D: Pediatric arteriovenous malformations: a 15-year experience with an emphasis on residual and recurrent lesions. Childs Nerv Syst 23:31-37, 2007

18. Kondziolka D, Humphreys RP, Hoffman HJ, Hendrick EB, Drake JM: Arteriovenous malformations of the brain in children: a forty year experience. Can J Neurol Sci 19:40-45, 1992

19. McKissock W, Paterson JH: A clinical survey of intracranial angiomas with special reference to their mode of progression and surgical treatment: a report of 110 cases. Brain 79:233-266, 1956

20. Nornes H, Lundar T, Wikeby P: Cerebral arteriovenous malformations; results of microsurgical management. Acta Neurochir (Wien) 50:243-257, 1979

21. Padget DH: The cranial venous system in man in reference to development, adult configuration, and relation to the arteries. Am J Anat 98:307-355, 1956

22. Park YS, Kwon JT: Recurrent cerebral arteriovenous malformation in a child: case report and review of the literature. $\mathbf{J}$ Korean Neurosurg Soc 45:401-404, 2009

23. Pellettieri L, Svendsen P, Wikholm G, Carlsson CA: Hidden compartments in AVMs-a new concept. Acta Radiol 38:2 7, 1997

24. Rodríguez-Arias C, Martínez R, Rey G, Bravo G: Recurrence in a different location of a cerebral arteriovenous malformation in a child after radiosurgery. Childs Nerv Syst 16:363365,2000

25. Sano K, Ueda Y, Saito I: Subarachnoid hemorrhage in children. Childs Brain 4:38-46, 1978

26. Santoro A, Giuliani S, Paolini S: Recurrent arteriovenous malformation in an adult. Case illustration. J Neurosurg 93:1082, 2000

27. Schmit BP, Burrows PE, Kuban K, Goumnerova L, Scott RM: Acquired cerebral arteriovenous malformation in a child with moyamoya disease. Case report. J Neurosurg 84:677-680, 1996

28. Sonstein WJ, Kader A, Michelsen WJ, Llena JF, Hirano A, Casper D: Expression of vascular endothelial growth factor in pediatric and adult cerebral arteriovenous malformations: an immunocytochemical study. J Neurosurg 85:838-845, 1996

29. Weil AG, Li S, Zhao JZ: Recurrence of a cerebral arteriovenous malformation following complete surgical resection: a case report and review of the literature. Surg Neurol Int 2:175-180, 2011

30. Wilson CB, Hoi Sang U, Domingue J: Microsurgical treatment of intracranial vascular malformations. J Neurosurg 51: 446-454, 1979

31. Yaşargil MG: Microneurosurgery: AVM of the Brain, Clinical Considerations, General and Special Operative Techniques, Surgical Results, Nonoperated Cases, Cavernous and Venous Angiomas, Neuroanesthesia. New York: Thieme, 1988, Vol III B

Manuscript submitted May 15, 2012.

Accepted August 1, 2012.

Portions of this work were presented in abstract form and in an oral presentation at the 16th Annual Leksell Gamma Knife Society meeting, March 29, 2012, in Sydney, Australia.

Please include this information when citing this paper: DOI: 10.3171/2012.8.GKS12994.

Address correspondence to: Do Hoon Kwon, M.D., Ph.D., Department of Neurological Surgery, Asan Medical Center, University of Ulsan, College of Medicine, 88 Olympic-ro 43 gil, Songpa-gu, Seoul 138-736, Korea. email: ykwon@amc.seoul.kr. 